\title{
Mobility of an in-stent plaque protrusion detected with carotid duplex during the carotid artery stenting procedure: a case report
}

\author{
Kanta Tanaka $^{1,2}$, Takamichi Kitagawa ${ }^{3}$, Norikazu Yamana ${ }^{4}$, Yoshinori Akiyama ${ }^{2,4}$ \\ ${ }^{1}$ Department of Neurology, ${ }^{2}$ Stroke Center, ${ }^{3}$ Department of Clinical Pathology, ${ }^{4}$ Department of Neurosurgery, Tenri \\ Hospital, Tenri, Japan
}

\begin{abstract}
In-stent plaque protrusion (ISP) is a predictor for ischemic complications in carotid artery stenting (CAS). Because its mobility would further increase the distal embolization risk, the intraprocedural detection of mobility is important but has not yet been reported. We present an 87-year-old male with symptomatic right carotid artery stenosis who underwent CAS. Intravascular ultrasound (IVUS) after stent deployment revealed a small ISP, and simultaneous extravascular carotid duplex disclosed its mobility. After coverage of the ISP with stent-in-stent implantation, no remaining protrusion was observed with either IVUS or carotid duplex. The patient experienced no periprocedural strokes. Carotid duplex is suited to evaluate targets' movement because of its excellent temporal resolution. Carotid duplex has potential value for intraprocedural risk estimation of ISP.

Keywords: carotid artery stenting; carotid duplex; in-stent plaque protrusion; mobility
\end{abstract}

\section{Introduction}

Carotid artery stenting (CAS) has become a noninferior alternative to carotid endarterectomy (CEA), particularly for patients who are at increased systemic or surgical risk [1-3]. However, the incidence of stroke complication is higher in CAS than in CEA; therefore, the reduction of the incidence of periprocedural ischemic stroke is mandatory to improve the safety of CAS $[2,4]$. An important feature that discriminates CAS from CEA is the presence of residual plaque outside the stent struts, which may protrude into the vascular lumen via the stent cells. This in-stent plaque protrusion (ISP) is a potential predictor for ischemic complications in CAS [5]. However, to date, the characteristics of ISP for determining the indication of an additional treatment to prevent distal

Received 19.04.2018 Accepted 08.06.2018

Med Ultrason

2019, Vol. 21, No 1, 89-92

Corresponding author: Kanta Tanaka

Department of Neurology, Tenri Hospital, 200 Mishima, Tenri, Nara 632-8552, Japan Phone: +81743635611

E-mail: tanaka19830311kanta@gmail.com embolization have not been reported. Although mobility has potential as an important feature of high-risk ISP, there have been no reports about it.

To date, several studies have reported the usefulness of adjunctive carotid duplex in CAS, particularly in minimizing iodinated contrast usage [6-8]. Here, we present the case of a patient with ISP with mobile features, which was detected using extravascular ultrasonography during the procedure of duplex-assisted CAS without the use of a contrast medium. Our aim is to report a potential additive value of carotid duplex for the improvement of CAS safety.

\section{Case report}

An 87-year-old male developed progressive left hemiparesis over a period of 3 days prior to admission. He had a history of dyspnea due to an allergy to an iodinated contrast. He had no history of chronic kidney disease, diabetes mellitus, or dyslipidemia. On admission, his left arm and left leg could be held at $45^{\circ}$ and $30^{\circ}$, respectively, in the supine position, but they slowly drifted downward. Diffusion-weighted imaging (DWI) demonstrated small acute infarctions at the 
edge of the right middle cerebral artery region (fig 1a). Magnetic resonance angiography revealed 60\% stenosis of the right internal carotid artery (ICA) (fig 1b). T1-weighted black blood imaging of the right carotid plaque revealed elevated signal intensity, thereby suggesting its fragility (fig 1c). Carotid duplex showed an ulcerated plaque at the right carotid bulbus. The plaque displayed hyperechogenicity with an acoustic shadow, which indicated the presence of calcification (fig 1d). No significant acceleration of the flow velocities was observed (peak systolic velocity $=115 \mathrm{~cm} / \mathrm{s}$ ). The patient was diagnosed as having a cerebral infarction due to atherosclerotic artery-to-artery embolization. Although he underwent medical treatment with aspirin, clopidogrel, argatroban, and atorvastatin, his left hemiparesis worsened because of a recurrent attack on day 2 after admission. DWI showed several newly appeared infarcts at the right middle cerebral artery territory. Because he experienced recurrent ischemic stroke under the intensive medical therapy, early intervention was required. Considering his allergy to iodinated contrast and the unstable nature of the responsible plaque, CEA was recommended. However, he desired a less invasive treatment, and therefore we planned a CAS that could be performed with the administration of local anesthesia.

On day 7 after admission carotid duplex-assisted CAS was performed without the administration of a contrast medium. Written informed consent regarding the procedure was obtained from the patient. Fluoroscopic imaging was performed using a biplane flat detector angiography unit (Artis Zee Biplane; Siemens, Erlangen, Germany). Carotid duplex was performed by an experienced sonographer using a $12-\mathrm{MHz}$ linear array transducer (Aplio 400; Toshiba, Japan). Intravascular ultrasonography (IVUS; Volcano S5 imaging system; Volcano Corporation, San Diego, CA, USA) was also used. After an 8-Fr sheath (Super Arrow-flex Sheath Introducer $45 \mathrm{~cm}$; Teleflex, Japan) was inserted through the right femoral artery, 5000 IU of heparin sodium was intravenously administered. An 8-Fr Simmons-curved guiding catheter (NEURO-EBU $83 \mathrm{~cm}$; Gadelius Medical K. K., Japan) was fluoroscopically placed into the innominate artery. A FilterWire EZ (Boston Scientific, Japan) was fluoroscopically guided into the right common carotid artery. Under duplex guidance, the FilterWire EZ crossed the lesion and advanced into the right ICA. The filter was opened under fluoroscopy in the distal extracranial right ICA. We determined stent size on the basis of the IVUS measurement of arterial diameters and lesion length and level. Using carotid duplex monitoring, a PROTÉGÉ RX $9 \times 60-\mathrm{mm}$ stent (Covidien, Japan) was positioned

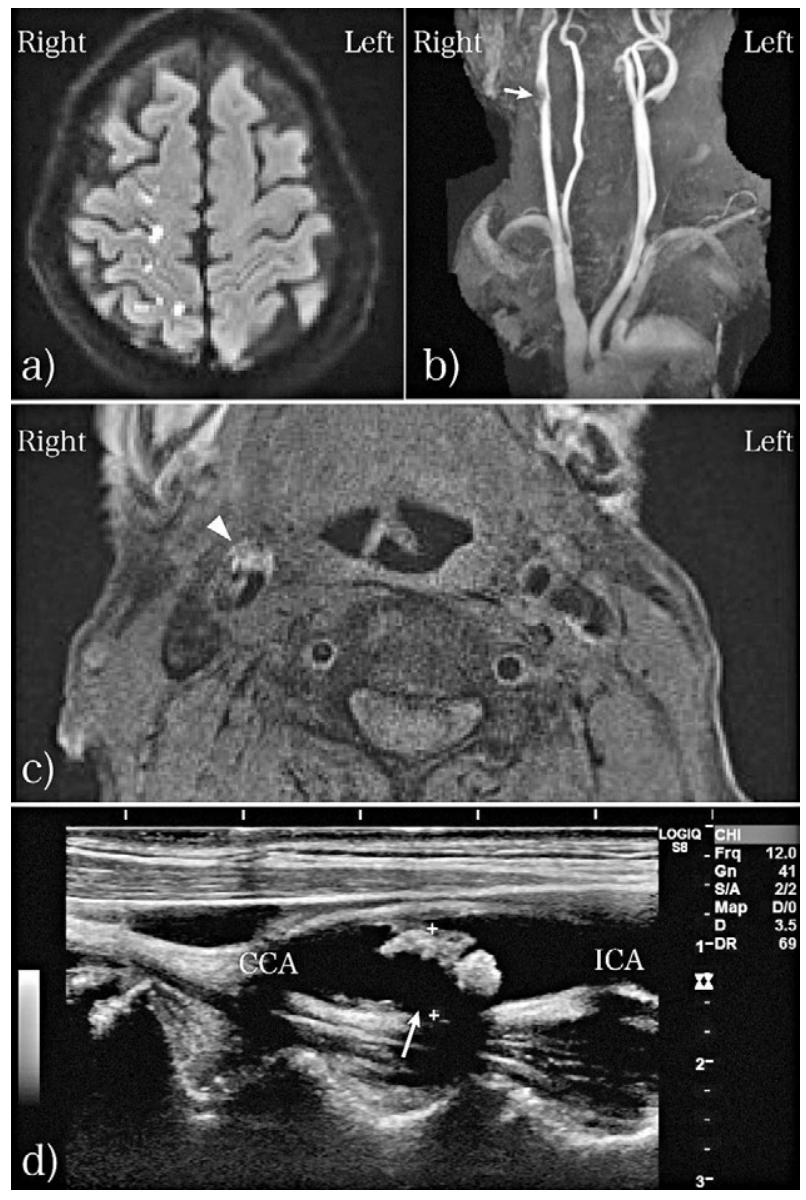

Fig 1. a) Diffusion-weighted imaging obtained at the time of admission showing multiple small acute infarctions over the right middle cerebral artery territory; b) Magnetic resonance angiography revealing $60 \%$ stenosis (arrow) in the right ICA and asymptomatic $70 \%$ stenosis in the left ICA; c) T1-weighted black blood imaging showing an elevated signal intensity (arrow head) in the right carotid plaque; d) Carotid ultrasonography using a $12-\mathrm{MHz}$ linear array transducer (LOGIQ S8; GE Healthcare, Japan) revealed an ulcerated plaque with a height of $7.4 \mathrm{~mm}$ at the right carotid bulbus (arrow). CCA, common carotid artery; ICA, internal carotid artery.

and deployed (fig 2a). Following this, IVUS revealed a small ISP with no distinct characteristics (fig 2b). We attempted to observe the ISP using extravascular carotid duplex, and the mobile features of the protrusion were disclosed (fig $2 \mathrm{c}-\mathrm{d}$ ). To prevent distal embolization, we performed stent-in-stent implantation (PRECISE PRO RX 9×30 mm stent; Cordis, Japan). No remaining protrusions were observed with either IVUS or carotid duplex (fig $2 \mathrm{e}-\mathrm{f}$ ), and the patient experienced no periprocedural strokes. Carotid duplex performed at 14 days and 6 months after the procedure showed no protrusion or restenosis. 


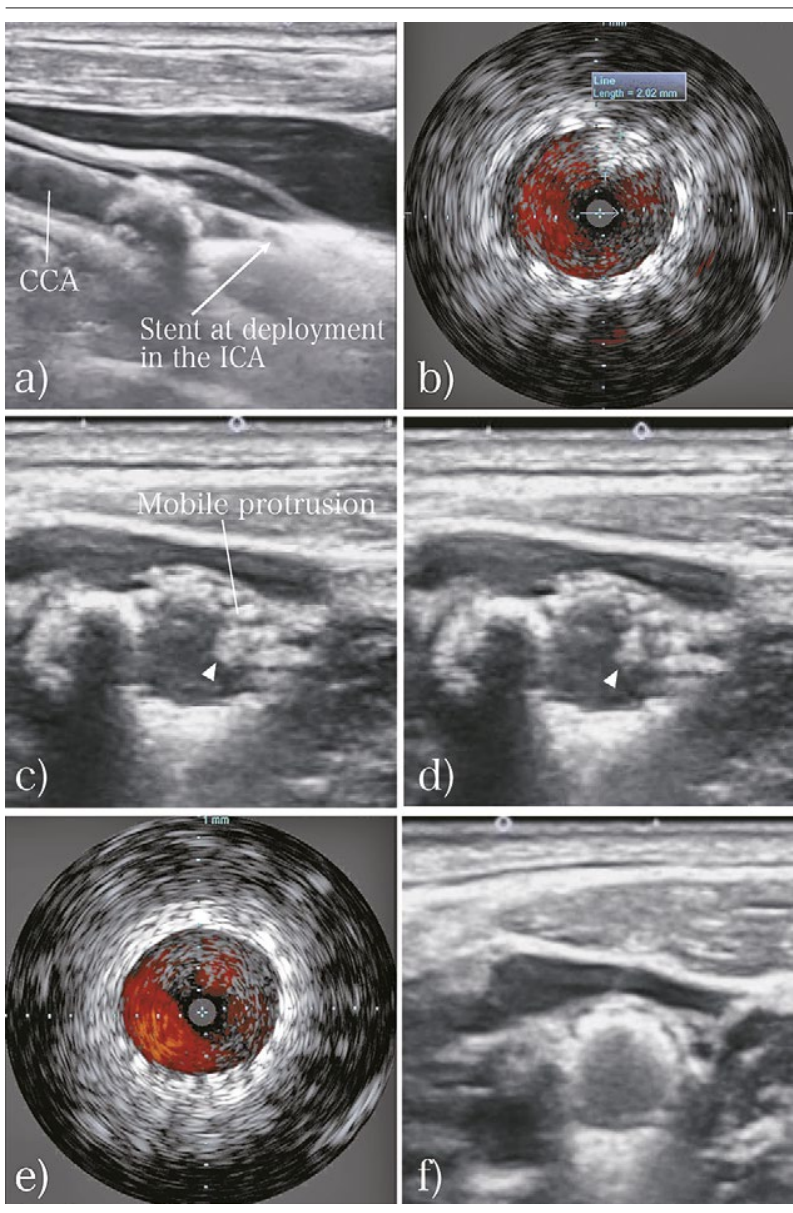

Fig 2. a) B-mode imaging of duplex-assisted CAS. A PROTÉGE RX $9 \times 60-\mathrm{mm}$ stent was delivered across the carotid plaque. Its deployment is proceeding. The deployed tip of the stent is depicted as a hyper-echoic object in the right ICA (arrow); b) IVUS revealing an ISP of approximately $2 \mathrm{~mm}$; c) and d) Bmode imaging revealing a small mobile mass (arrow heads), which bounced on the stent surface along with the heartbeat; e) and f) After coverage with stent-in-stent implantation, IVUS and duplex confirmed the disappearance of the protrusion. CAS, carotid artery stenting; CCA, common carotid artery; ICA, internal carotid artery; ISP, in-stent plaque protrusion; IVUS, intravascular ultrasonography.

\section{Discussions}

The novel and important finding in our patient was the mobility of an ISP revealed using the intraprocedural extravascular carotid duplex. Regarding carotid plaques encountered in ordinary clinical settings, their mobility is a well-known risk factor for strokes and carotid duplex is the modality of choice for its detection [9]. Because the mobility renders carotid plaques highly emboligenic, antithrombotic therapy alone is often insufficient to prevent recurrent strokes [10] and surgical removal (CEA) or coverage (CAS) of the mobile plaque is required [10-
12]. On the other hand, with respect to CAS-associated ISPs, there is no report on their mobility. However, it is plausible that the mobility of ISPs has a risk profile similar to the mobility of carotid plaques.

IVUS is a promising modality with high sensitivity for the detection of ISP [13]. IVUS can visualize even small ISPs because of its good spatial resolution. However, its use is usually not aimed at detecting the mobility of targets. In particular, if the size of ISP is small as is the case in our patient, then the detection of its mobility by IVUS may become difficult. In contrast, carotid duplex, which has the benefit of excellent temporal resolution, is suitable for visualizing the mobility of ISP [14]. Therefore, carotid duplex can not only be used as an adjunctive imaging technique for reducing or avoiding the use of a contrast medium [6-8] but also can be used to complement IVUS for the characterization of ISPs. Radiation exposure of a sonographer, which seems to be a major concern, can be prevented by stopping fluoroscopic imaging during carotid duplex scanning.

In conclusion, carotid duplex has a potential value for the intraprocedural risk estimation of ISP and will be useful for the adequate management of ISPs to improve the safety of CAS. However, further studies are warranted.

\section{Acknowledgement}

The authors would like to thank Dr. Toshihiko Suenaga and Dr. Hiroshi Yamagami for their academic support.

\section{References}

1. Yadav JS, Wholey MH, Kuntz RE, et al. Protected carotidartery stenting versus endarterectomy in high-risk patients. N Engl J Med 2004;351:1493-1501.

2. Brott TG, Hobson RW 2nd, Howard G, et al. Stenting versus endarterectomy for treatment of carotid-artery stenosis. N Engl J Med 2010;363:11-23.

3. Rosenfield K, Matsumura JS, Chaturvedi S, et al. Randomized trial of stent versus surgery for asymptomatic carotid stenosis. N Engl J Med 2016;374:1011-1020.

4. Carotid Stenting Trialists' Collaboration, Bonati LH, Dobson J, et al. Short-term outcome after stenting versus endarterectomy for symptomatic carotid stenosis: a preplanned meta-analysis of individual patient data. Lancet 2010;376:1062-1073.

5. Beppu M, Mineharu Y, Imamura H, et al. Postoperative instent protrusion is an important predictor of perioperative ischemic complications after carotid artery stenting. J Neuroradiol 2018 Mar 7. doi:10.1016/j.neurad.2018.02.009.

6. Ascher E, Hingorani AP, Marks N. Duplex-assisted internal carotid artery balloon angioplasty and stent placement. Perspect Vasc Surg Endovasc Ther 2007;19:41-47. 
7. Varcoe RL, Nammuni I, Lennox AF, Yang JL, Crowe P, Walsh WR. Adjunctive ultrasonography to minimize iodinated contrast administration during carotid artery stenting: a randomized trial. J Endovasc Ther 2012;19:638-647.

8. Mizowaki T, Fujita A, Imahori T, et al. Duplex-assisted carotid artery stenting without administration of contrast medium for patients with chronic kidney disease or allergic reaction. Neuroradiology 2016;58:679-686.

9. Ogata T, Yasaka M, Wakugawa Y, Kitazono T, OkadaY. Morphological classification of mobile plaques and their association with early recurrence of stroke. Cerebrovasc Dis 2010;30:606-611.

10. Delgado MG, Vega P, Roger R, Bogousslavsky J. Floating thrombus as a marker of unstable atheromatous carotid plaque. Ann Vasc Surg 2011;25:1142.e11-e17.
11. Funaki T, Iihara K, Miyamoto S, Nagatsuka K, Hishikawa T, Ishibashi-Ueda H. Histologic characterization of mobile and nonmobile carotid plaques detected with ultrasound imaging. J Vasc Surg 2011;53:977-983.

12. Oomura M, Sato C, Yamada K, Ikeda T, Anan C, Kamimoto $\mathrm{K}$. Carotid artery stenting successfully prevents progressive stroke due to mobile plaque. Case Rep Neurol 2015;7:121126.

13. Shinozaki N, Ogata N, Ikari Y. Plaque protrusion detected by intravascular ultrasound during carotid artery stenting. $\mathrm{J}$ Stroke Cerebrovasc Dis 2014;23:2622-2625.

14. Alexandrov AV. Practice of ultrasound: an introduction. In: Alexandrov AV. (ed). Cerebrovascular ultrasound in stroke prevention and treatment, 2nd Edition, Wiley-Blackwell, 2011: xiii. 